LAPTH-848/01

DTP-MSU/01-10

\title{
Conformal mechanics on rotating Bertotti-Robinson spacetime
}

\author{
Gérard Clément ${ }^{a}{ }^{*}$ and Dmitri Gal'tsov ${ }^{a, b} \dagger$ \\ ${ }^{a}$ Laboratoire de Physique Théorique LAPTH (CNRS), \\ B.P.110, F-74941 Annecy-le-Vieux cedex, France \\ ${ }^{b}$ Department of Theoretical Physics, \\ Moscow State University, 119899, Moscow, Russia,
}

23 May 2001

\begin{abstract}
We investigate conformal mechanics associated with the rotating Bertotti-Robinson (RBR) geometry found recently as the near-horizon limit of the extremal rotating Einstein-Maxwell-dilaton-axion black holes. The solution breaks the $S L(2, R) \times$ $S O(3)$ symmetry of Bertotti-Robinson (BR) spacetime to $S L(2, R) \times U(1)$ and breaks supersymmetry in the sense of $N=4, d=4$ supergravity as well. However, it shares with BR such properties as confinement of timelike geodesics and discreteness of the energy of test fields on the geodesically complete manifold. Conformal mechanics governing the radial geodesic motion coincides with that for a charged particle in the BR background (a relativistic version of the De Alfaro-Fubini-Furlan model), with the azimuthal momentum playing the role of a charge. Similarly to the BR case, the transition from Poincaré to global coordinates leads to a redefinition of the Hamiltonian making the energy spectrum discrete. Although the metric does not split into a product space even asymptotically, it still admits an infinitedimensional extension of $S L(2, R)$ as asymptotic symmetry. The latter is shown to be given by the product of one copy of the Virasoro algebra and $U(1)$, the same being valid for the extremal Kerr throat.
\end{abstract}

PACS no: 04.20.Jb, 04.50.+h, 46.70.Hg

*Email: gclement@lapp.in2p3.fr

†Email: galtsov@grg.phys.msu.su 


\section{Introduction}

In the chain of $A d S_{n} / C F T_{(n-1)}$ dualities [1] the lowest case $n=2$ attracted recently much attention. The underlying conformal theory is one-dimensional quantum mechanics, which is intimately connected with two-dimensional CFT (dual to $A d S_{3}$ ), from which it inherits a Virasoro extension. While higher- $n$ dualities are mostly interesting in what they teach us about (flat space) gauge theories, the $n=2$ case is interesting in its gravitational aspects - two-dimensional gravity, black hole entropy, etc. From the 'brane' viewpoint, the $A d S_{2}$ geometry is encountered in the throat limit of an extremal Reissner-Nordström black hole which is given by the direct product $A d S_{2} \times S^{2}$ known as the Bertotti-Robinson (BR) spacetime. The former is a $1 / 2$ BPS state of $N=2$ four-dimensional supergravity [2], 3], whilst the throat exhibits the full $N=2 \mathrm{SUSY}$. The $A d S_{2}$ sector is encountered also in several intersecting branes of ten and eleven-dimensional supergravities [4] and $D 0$ branes in any dimensions [5, 6].

The $S L(2, R)$ isometry group of $A d S_{2}$ is the conformal group on a line. One way to uncover the conformal mechanics associated with BR is to analyse radial motion of neutral or charged particles down the throat. It is appropriate to distinguish the $A d S_{2}^{0}$ patch which covers only the exterior part of the throat from the full geodesically complete $A d S_{2}$ space, which we call here $A d S_{2}^{+}$. Geodesic motion on $A d S_{2}^{0}$ was shown [7, 8] to be related to the relativistic version of the De Alfaro-Fubini-Furlan (DFF) conformal mechanics [9]. The DFF hamiltonian has a continuous positive semi-definite spectrum and no zero energy ground state. As was shown in [10], passing to $A d S_{2}^{+}$is equivalent to adding a potential term making the spectrum discrete with a well-defined ground state. The DFF model may be regarded as a two-particle Calogero model, and it was suggested by Gibbons and Townsend [10] that the $N=4$ superextension of the large $n$-particle Calogero model may describe quantum extreme RN black holes. The related superconformal models were discussed in [11, 12, 13, 14]. An alternative way to reach an infinite-dimensional extension of the conformal mechanics consists in revealing a 'hidden' Virasoro symmetry, this may be achieved both non-geometrically [15, 16, 17, 18, 19] and by exploring the asymptotic symmetry of BR (see below). Still the problem of a holographic description of microscopic degrees of freedom of extreme RN black holes remains open, which is perhaps related to the existence of the mass gap [20] excluding non-singular classical deformations of spacetime. An alternative interpretation of internal degrees of freedom of extremal RN black holes was suggested in [21, 22, 23] as 'fragmentation' of the throat.

A natural arena to explore the $A d S_{2} / C F T_{1}$ correspondence is two-dimensional gravity 24, 25, 26, 27, 28, 29, 30, 31, 32, 33, 34. The major physical question is related to the entropy of two-dimensional black holes. The analysis starts with revealing that the asymptotic symmetry of $A d S_{2}$ is given by the Virasoro algebra acting on a line instead of the two-dimensional boundary of $A d S_{3}$. In this latter case, as was shown by Brown and Henneaux [35], the deformations of spacetime preserving the structure of the asymptotic boundary form a projective representation of the Virasoro symmetry with a (classically calculable) central charge (for a more recent derivation using path integrals see [36]). The definition of the corresponding algebra of charges for $A d S_{2}$ is more subtle since the boundary of a one-dimensional manifold has zero dimensionality. A way out was suggested by Cadoni and Mignemi 25] by introducing some time average for the charges. Using this 
procedure the central charges for $A d S_{2}$ were obtained in [25] and [26] (with somewhat different parameterization for spacetime deformations) differing by a factor of two (for a detailed discussion see [27]). The central charge is presumably related to the entropy of two-dimensional black holes via Cardy's formula (as was suggested earlier by Strominger for the BTZ black hole [37]). The correct match is straightforward in the case of the value found in [26], but requires accounting for the entanglement entropy due to the second component of the boundary of $A d S_{2}$ [31] to obtain the value of [25]. For a discussion of this discrepancy see also [28, 32, 33]. The relevance of the $2 d$ gravity central charge to the entropy of the extreme four-dimensional RN black hole is problematic, though it may be responsible for a variation of the entropy for a charged black hole away from extremality [20, 26].

One can hope to make further progress in understanding the puzzles of $A d S_{2} / C F T_{1}$ by exploring other backgrounds with $A d S_{2}$ sectors. It was suggested by Bardeen and Horowitz [38] (BH) that the near-horizon geometry of rotating four-dimensional black holes (for an earlier discussion see [39]) can also provide an arena for holography. The isometry group of the Kerr throat is $S L(2, R) \times U(1)$, where $U(1)$ is a remnant of the spherical symmetry of BR. Although the geometry can not be presented globally as a direct product of $A d S_{2}$ with anything, it still preserves some typical AdS properties. The main difference with higher-dimensional rotating brane/black hole spaces 20, 40, 41, 42, 43] is that for the four-dimensional Kerr spacetime the AdS sector does not factor out even asymptotically. (However, a counterexample of a five-dimensional $A d S_{5}$ Einstein-Maxwell black hole was constructed recently [44]). Still the Kerr throat geometry turned out to be too complicated for analysis, so no holographic dual was found in [38]. In particular, it inherits from the full Kerr spacetime the superradiance phenomenon whose interpretation in the holographic context is obscure.

Here we make some progress in this direction using a geometry which possesses the same isometry $S L(2, R) \times U(1)$ as the $\mathrm{BH}$ solution but does not contain the angular factors causing the above complications. Our solution is non-vacuum, it is supported by a vector field and a pseudoscalar axion. This geometry was previously found as a particular near horizon limit of rotating dilaton-axion black holes 445. Here we show that it is an exact solution of the four-dimensional Einstein-Maxwell-dilaton-axion theory, which is a consistent truncation of the $N=4, d=4$ supergravity or an effective heterotic string theory. Then we investigate properties underlying the structure of the dual conformal mechanics. We show that confinement of geodesics is manifest exactly in the same way as in the BR case. The same is true for the frequency spectrum of the test scalar field on the geodesically complete manifold: the spectrum is discrete and there is no superradiance. Analyzing the radial part of the wave equation we find that for the $R B R^{0}$ coordinate patch the underlying conformal mechanics is again the relativistic De Alfaro-Fubini-Furlan model found in [7] for the BR case, with the angular momentum playing the role of the electric charge of [7]. So the spectrum is continuous with zero energy excluded. On the contrary, the spectrum is discrete and bounded from below if one deals with the full geodesically complete manifold. We construct explicitly the spectrum generated algebra related to the conformal symmetry.

The conformal boundary of the rotating BR is a three-dimensional spacetime which, unlike BR, does not factorise globally into the product of a line and a 2- sphere. Moreover, 
the conformally rescaled boundary is now singular. We show that in spite of this the asymptotic symmetry of RBR is infinite dimensional and is given by the product of (one copy of) the Virasoro algebra (without a central charge) with a circle. The same holds for the near-horizon geometry of the extremal Kerr-Newman black hole. The generators of the infinite symmetry contain azimuthal derivatives, which reflect the rotating nature of the spacetime.

The plan of the paper is as follows. In section 2 we review our new solution as well as some related ones and discuss isometries and various coordinate patches. In section 3 the geodesics and the modes of a test scalar field are analysed on the full RBR spacetime. We show that all timelike geodesics are confined while the equatorial null ones can escape to infinity but in an infinite time. So the RBR spacetime is geodesically complete. The frequencies of the Klein-Gordon modes are discrete and form an equidistant spectrum. In section 4 an associated conformal mechanics is presented. Section 5 is devoted to asymptotic symmetries of both BR and RBR spacetimes. We conclude in 6 with some general remarks.

\section{RBR solution to dilaton-axion gravity}

We start by recalling the Bertotti-Robinson (BR) metric which is a solution to the fourdimensional Einstein-Maxwell theory

$$
d s^{2}=-r^{2} d \tau^{2}+\frac{d r^{2}}{r^{2}}+d \theta^{2}+\sin ^{2} \theta d \varphi^{2}
$$

supported by the Maxwell field

$$
A=q r d \tau+p \cos \theta d \varphi
$$

where electric and magnetic charges are subject to the condition $q^{2}+p^{2}=1$ according to the choice of the unit radius of the hyperboloid. This solution is known to represent the near-horizon geometry of the extreme Reissner-Nordstrom (RN) black holes and is a fully supersymmetric solution to $D=4, \mathcal{N}=2$ supergravity [2, 3]. Alternatively, (2.1) arises in the Kaluza-Klein reduction of a five-dimensional vacuum solution with zero dilaton (i.e. with equal electric and magnetic strengths in four dimensions) 45.

Geometrically this is a product space $A d S_{2} \times S^{2}$, whose AdS component can be viewed as due to the effective cosmological constant in two dimensions resulting from the Maxwell field. The coordinates (2.1) cover only a half of $A d S_{2}$, but can be continued to the full manifold via an appropriate coordinate transformation. Namely, the one-parameter family of metrics

$$
d s^{2}=-\left(x^{2}+b\right) d t^{2}+\frac{d x^{2}}{x^{2}+b}+d \theta^{2}+\sin ^{2} \theta d \phi^{2},
$$

satisfies the Einstein-Maxwell equations with the potential $A=q x d \tau+p \cos \theta d \phi$ for any real $b$. For $b>0$ the domain of $x$ (initially $r \geq 0$ ) can be extended to the full real line thus covering the entire $A d S_{2}$ manifold (for $b \leq 0$ the radial coordinate is restricted to $x \geq \sqrt{-b}$ ). To describe different coordinate patches on $A d S_{2}$ it is enough to consider $b=0, \pm 1$, we will call the corresponding metrics as $B R^{0}, B R^{ \pm}$(our notation for the last 
two is opposite to the one used in [25]). The $B R^{+}$space is geodesically complete, while $B R^{0}, B R^{-}$have a Killing horizon at $x=0,1$ respectively.

Bardeen and Horowitz [38] (BH) observed that an effective cosmological constant in two dimensions may also arise from the Kaluza-Klein two-form in dimensional reduction of a four-dimensional vacuum metric, namely the extremal rotating Kerr black hole. It has been shown that the near-horizon geometry of the Kerr black hole contains indeed the $A d S_{2}$ sector, though the direct product structure of the four-dimensional spacetime is lost:

$$
d s^{2}=\frac{1}{2}\left(1+\cos ^{2} \theta\right)\left[-r^{2} d \tau^{2}+\frac{d r^{2}}{r^{2}}+d \theta^{2}\right]+\frac{2 \sin ^{2} \theta}{1+\cos ^{2} \theta}(d \varphi+r d \tau)^{2} .
$$

It can be checked this this metric is an exact solution of the vacuum Einstein euqations possessing an isometry group $S L(2, R) \times U(1)$. It approaches $A d S_{2} \times S^{2}$ near the polar axis for small $r$, but for larger $r$ the non-diagonal $\phi-\tau$ term becomes manifest. Here the domain of $r$ is also a semi-axis, and there is a Killing horizon at $r=0$. The Killing vector $\partial_{\tau}$ becomes spacelike inside the ergosphere which is given by

$$
\cos 2 \theta<4 \sqrt{3}-7
$$

By coordinate transformation one can rewrite the solution in a form similar to (2.3):

$$
d s^{2}=\frac{1}{2}\left(1+\cos ^{2} \theta\right)\left[-\left(x^{2}+b\right) d t^{2}+\frac{d x^{2}}{x^{2}+b}+d \theta^{2}\right]+\frac{2 \sin ^{2} \theta}{1+\cos ^{2} \theta}(d \phi+x d t)^{2},
$$

for $b>0$ this patch covers the entire hyperboloid. Similarly to the case of AdS, we will label the three distinct patches $b=0, \pm 1$ of the $\mathrm{BH}$ solution as $B H^{0, \pm}$. It was shown in [38] that $\mathrm{BH}^{+}$partly preserves such typical AdS features as confinement of timelike geodesics and discrete energy spectrum of a minimally coupled test scalar field. However, the presence of $\theta$-dependent factors violates this simple picture: a class of geodesics can escape to infinity, and the Klein-Gordon spectrum also contains a continuous part with an associated superradiance phenomenon. Note that the $\mathrm{BH}$ spacetime does not admit Killing spinors.

Recently we have found 45] another spacetime which which lies between (2.1) and (2.4): the metric is non-diagonal but does not contain $\theta$-factors. The price of this is its non-vacuum nature, the solution was obtained as the limit $M \rightarrow \infty, a \rightarrow 0(J=M a$ fixed) of the near-horizon limit of extremal rotating dilaton-axion black holes [46]. The action of this theory, which is a truncated bosonic sector of $D=4, \mathcal{N}=4$ supergravity or toroidally compactified heterotic string, describes the gravity-coupled system of two scalar fields (dilaton $\Phi$ and (pseudoscalar) axion $\kappa$ ), and one Abelian vector field $A_{\mu}$, :

$$
S=\frac{1}{16 \pi} \int d^{4} x \sqrt{|g|}\left\{-R-2 \partial_{\mu} \Phi \partial^{\mu} \Phi-\frac{1}{2} e^{4 \Phi} \partial_{\mu} \kappa \partial^{\mu} \kappa-e^{-2 \Phi} F_{\mu \nu} F^{\mu \nu}-\kappa F_{\mu \nu} \tilde{F}^{\mu \nu}\right\},
$$

where円 $\tilde{F}^{\mu \nu}=\frac{1}{2} E^{\mu \nu \lambda \tau} F_{\lambda \tau}, F=d A$. Our new metric looks like a 'pure' rotating BR:

$$
d s^{2}=-r^{2} d \tau^{2}+\frac{d r^{2}}{r^{2}}+d \theta^{2}+\sin ^{2} \theta(d \phi+r d \tau)^{2}
$$

${ }^{1}$ Here $E^{\mu \nu \lambda \tau} \equiv|g|^{-1 / 2} \varepsilon^{\mu \nu \lambda \tau}$, with $\varepsilon^{1234}=+1$, where $x^{4}=t$ is the time coordinate. 
which coincides with (2.4) near the polar axis. This geometry is supported by a non-zero Maxwell field which is the radial magnetic field distorted by the rotation induced Faraday effect

$$
A=A_{\mu} d x^{\mu}=-\frac{\cos \theta}{\sqrt{2}}(d \phi+r d \tau)
$$

and by the linear axion

$$
\kappa=\cos \theta
$$

the dilaton field being identically constant (set to zero). The normalization corresponds to the chosen unit radius of $A d S_{2}$.

First we want to demonstrate that this limiting solution is an exact solution of the field equations following from the action (2.7). The non-zero components of the Einstein tensor $G_{\mu \nu}=R_{\mu \nu}-g_{\mu \nu} R / 2$ in the orthonormal frame (denoted by barred indices) read

$$
G_{\bar{\tau} \bar{\tau}}=-G_{\bar{r} \bar{r}}=G_{\bar{\theta} \bar{\theta}}=\frac{1}{4}\left(3+\cos ^{2} \theta\right), \quad G_{\bar{\phi} \bar{\phi}}=\frac{1}{4}\left(3 \cos ^{2} \theta+1\right),
$$

while the components of the Maxwell stress-tensor (multiplied by $8 \pi$ ) are

$$
T_{\bar{\tau} \bar{\tau}}=-T_{\bar{r} \bar{r}}=T_{\bar{\theta} \bar{\theta}}=T_{\bar{\phi} \bar{\phi}}=\frac{1}{4}\left(\cos ^{2} \theta+1\right),
$$

the mismatch being accounted by the axion stress-tensor

$$
T_{\bar{\tau} \bar{\tau}}=-T_{\bar{r} \bar{r}}=T_{\bar{\theta} \bar{\theta}}=-T_{\bar{\phi} \bar{\phi}}=\frac{1}{4} \sin ^{2} \theta .
$$

It is simple to check the validity of the Maxwell equations for the EMDA theory

$$
\nabla_{\nu}\left(F^{\mu \nu}+\kappa \tilde{F}^{\mu \nu}\right)=0
$$

and the axion equation

$$
\square \kappa=2 F_{\mu \nu} \tilde{F}^{\mu \nu},
$$

(these are written for a vanishing dilaton field), as well as the compensation of the two right-hand side terms in the dilaton equation

$$
2 \square \phi=-F_{\mu \nu} F^{\mu \nu}+(\nabla \kappa)^{2} .
$$

Alternatively, as was shown in [45], the metric (2.8) can be regarded as originating from five-dimensional gravity coupled to dilaton and axion, or from six-dimensional vacuum gravity. More recently, a similar solution was obtained in the five-dimensional EinsteinMaxwell theory with cosmological constant [44.

Both (2.4) and our solution break the $S O(3)$ symmetry of (2.1). The remaining isometry group $S L(2, R) \times U(1)$ is generated by the four Killing vectors

$$
\begin{aligned}
L_{0} & =\tau \partial_{\tau}-r \partial_{r}, \\
L_{1} & =\partial_{\tau}, \\
L_{-1} & =\left(r^{-2}+\tau^{2}\right) \partial_{\tau}-2 r \tau \partial_{r}-2 r^{-1} \partial_{\phi}, \\
L_{\phi} & =\partial_{\phi} .
\end{aligned}
$$


The first three of these generate the $\operatorname{sl}(2, R)$ algebra

$$
\left[L_{n}, L_{m}\right]=(n-m) L_{n+m}, \quad m, n=0, \pm 1 .
$$

which is an isometry of $A d S_{2}$ space (and a conformal symmetry of the line). However the four-dimensional spacetime is not a direct product of $A d S_{2}$ with another two-dimensional space, as reflected in the presence of the term $\partial_{\phi}$ in $L_{-1}$.

An attractive feature shared by both metrics (2.8) and (2.4) is that the conformal boundary at infinity is a timelike surface. Contrary to the BR case, however, the conformal boundary is now a singular curved space-time. Writing the metric as $d s^{2}=r^{2} d s_{c}^{2}$, one finds for (2.8):

$$
d s_{c}^{2}=-d \tau^{2}+d \rho^{2}+\rho^{2}\left[d \theta^{2}+\sin ^{2} \theta\left(d \phi+\frac{d \tau}{\rho}\right)^{2}\right],
$$

where $\rho=1 / r$. The scalar curvature of this spacetime

$$
R_{c}=\frac{3 \sin ^{2} \theta}{2 \rho^{2}}
$$

diverges on the boundary $\rho \rightarrow 0$. Nevertheless, as we will see below, many features of the BR spacetime which are relevant for $A d S / C F T$ correspondence still hold in our case.

The variables $\tau, r$ are Poincaré coordinates on $A d S_{2}$ which cover only half of the AdS hyperboloid in three-dimensional spacetime. Global coordinates are introduced via the change of coordinates

$$
r=\sqrt{1+x^{2}} \cos t+x, \quad r \tau=\sqrt{1+x^{2}} \sin t
$$

transforming

$$
-r^{2} d \tau^{2}+\frac{d r^{2}}{r^{2}}=-\left(1+x^{2}\right) d t^{2}+\frac{d x^{2}}{1+x^{2}} .
$$

In addition, the angular coordinate transformation

$$
\phi=\varphi+\ln \left|\frac{\cos t+x \sin t}{1+\sqrt{1+x^{2}} \sin t}\right|
$$

preserves the angular term

$$
d \phi+r d \tau=d \varphi+x d t
$$

Applying this to our initial $R B R^{0}$ solution (2.8) we obtain the geodesically complete metric $R B R^{+}$

$$
d s^{2}=-\left(1+x^{2}\right) d t^{2}+\frac{d x^{2}}{1+x^{2}}+d \theta^{2}+\sin ^{2} \theta(d \varphi+x d t)^{2},
$$

or, in a more symmetric form with $y=\cos \theta$,

$$
d s^{2}=-\left(1+x^{2}\right) d t^{2}+\frac{d x^{2}}{1+x^{2}}+\frac{d y^{2}}{1-y^{2}}+\left(1-y^{2}\right)(d \varphi+x d t)^{2}
$$


This metric cover the full $A d S_{2}$ hyperboloid. Note that its boundary has two disconnected components $x \rightarrow \pm \infty$, as in the $A d S_{2}$ case (contrary to higher $n \operatorname{AdS}$ ).

Another useful coordinate transformation

$$
r=\sqrt{x^{2}-1} \cosh t+x, \quad r \tau=\sqrt{x^{2}-1} \sinh t, \quad \phi=\varphi+\ln \left|\frac{\cosh t+x \sinh t}{1+\sqrt{x^{2}-1} \sinh t}\right|
$$

takes the interval into the $R B R^{-}$form

$$
d s^{2}=-\left(x^{2}-1\right) d t^{2}+\frac{d x^{2}}{x^{2}-1}+\frac{d y^{2}}{1-y^{2}}+\left(1-y^{2}\right)(d \varphi+x d t)^{2},
$$

with $x \geq 1$ and a Killing horizon at $x=1$.

These three coordinate patches, together with their rescalings, may be combined in the following solution valid for any real $b$ :

$$
\begin{aligned}
d s^{2} & =-\left(x^{2}+b\right) d t^{2}+\frac{d x^{2}}{x^{2}+b}+\frac{d y^{2}}{1-y^{2}}+\left(1-y^{2}\right)(d \varphi+x d t)^{2} \\
A & =-\frac{y}{\sqrt{2}}(d \varphi+x d t), \quad \phi=0, \quad \kappa=-y
\end{aligned}
$$

where we have taken care that the coordinate transformation $(r, \theta, \varphi, t) \rightarrow(x, y, \varphi, t)$ reverses orientations $(\partial y / \partial \theta=-\sin \theta<0)$ so that the sign of the pseudoscalar $\kappa$ must be reversed when going from (2.10) to (2.29).

Note that for $R B R^{-}(b=-1)$ one can make the complex transformation

$$
t \leftrightarrow i \varphi, \quad x \leftrightarrow-y, \quad d s^{2} \rightarrow-d s^{2}, \quad A \rightarrow-i A
$$

leading to the Bertotti-Robinson-NUT solution:

$$
\begin{aligned}
d s_{4}^{2} & =-\left(x^{2}-1\right)(d t+y d \varphi)^{2}+\frac{d x^{2}}{x^{2}-1}+\frac{d y^{2}}{1-y^{2}}+\left(1-y^{2}\right) d \varphi^{2} \\
A & =\frac{x}{\sqrt{2}}(d t+y d \varphi), \quad \phi=0, \quad \kappa=x .
\end{aligned}
$$

This solution breaks the $S L(2, R)$ symmetry of $A d S_{2} \times S^{2}$, but preserves the spherical symmetry, the isometry group being $U(1) \times S O(3)$.

All the solutions obtained from the extremal rotating EMDA black holes [46] are non supersymmetric in the context of $N=4, d=4$ supergravity 445. This is perhaps not surprising since these black holes are not BPS states. Non-rotating (and NUT-less) BPS EMDA black holes have vanishing horizon radius, while here we used black holes whose horizon radius remains finite in the extremal limit due to rotation. Note, however, that the isometry group $S L(2, R) \times U(1)$ has a natural superextension $S U(1,1 \mid 1)$.

\section{Geodesics and test scalar field on $\mathrm{RBR}^{+}$}

To probe the geometry (2.25) of a maximally extended patch let us consider geodesics for a particle of mass $\mu$ (possibly zero). The Hamilton-Jacobi equation in the RBR 
spacetime fully separates, and the geodesic equations can be integrated explicitly. The Killing vectors $L_{1}$ and $L_{\phi}$ also apply to $R B R^{+}$(2.25) (in terms of $t, \varphi$ ), generating the conserved energy and the azimuthal component of the momentum

$$
\mathcal{E}=-\dot{x}^{\nu} g_{\nu t}, \quad L=\dot{x}^{\nu} g_{\nu \varphi}
$$

where dots denote derivatives with respect to the affine parameter $\lambda$ on the geodesics. The constraint equation $g_{\mu \nu} \dot{x}^{\mu} \dot{x}^{\nu}=\varepsilon$ with $\varepsilon=1$ for timelike and $\varepsilon=0$ for null geodesics, reads

$$
\left(x^{2}+1\right)^{-1}\left(\dot{x}^{2}-(\mathcal{E}+x L)^{2}\right)+L^{2} \sin ^{-2} \theta+\dot{\theta}^{2}+\varepsilon=0 .
$$

This separates to the $\theta$-equation

$$
\dot{\theta}^{2}+L^{2} \sin ^{-2} \theta=\mathcal{K}^{2}
$$

where the separation constant $\mathcal{K}^{2} \geq L^{2}$ at the right hand side has the meaning of the total angular momentum, and the radial equation

$$
\dot{x}^{2}+\left(\mathcal{K}^{2}+\varepsilon\right)\left(1+x^{2}\right)-(\mathcal{E}+x L)^{2}=0
$$

Integration of (3.3) gives

$$
\cos \theta=\sqrt{1-L^{2} / \mathcal{K}^{2}} \cos (K \lambda)
$$

while integration of (3.4)

$$
x=\frac{\mathcal{E} L}{q^{2}}+a \cos q \lambda
$$

where

$$
q^{2}=\mathcal{K}^{2}-L^{2}+\varepsilon, \quad a^{2} q^{4}=\left(\mathcal{K}^{2}+\varepsilon\right)\left(\mathcal{E}^{2}-q^{2}\right) .
$$

Geodesics remain confined, unless $q=0$, which happens when

$$
\varepsilon=0, \quad \mathcal{K}^{2}=L^{2} .
$$

This corresponds to the equatorial propagation $(\theta=\pi / 2)$ of a massless particle. Coming back to Eq. (3.4) we find in this case

$$
\lambda=\int \frac{d x}{\sqrt{\mathcal{E}^{2}-L^{2}+2 x \mathcal{E} L}}
$$

From this equation it is clear that null geodesics can escape to infinity, but only for infinite values of the affine parameter. So the spacetime is geodesically complete.

Let us now investigate the modes of a minimally coupled scalar field. The inverse metric for (2.25) reads

$$
g^{\nu \lambda} \partial_{\nu} \partial_{\lambda}=\frac{1}{1+x^{2}}\left(-\partial_{t}^{2}+\frac{1-x^{2} \cos ^{2} \theta}{\sin ^{2} \theta} \partial_{\varphi}^{2}+2 x \partial_{t} \partial_{\varphi}\right)+\left(1+x^{2}\right) \partial_{x}^{2}+\partial_{\theta}^{2},
$$


while $\sqrt{-g}=\sin \theta$, so that the Klein-Gordon equation for the modes $\psi=\psi(x, \theta) e^{i(m \varphi-\omega t)}$ takes the form

$$
\begin{gathered}
\frac{1}{\sqrt{-g}} \partial_{\nu}\left(\sqrt{-g} g^{\nu \lambda} \partial_{\lambda} \psi\right)=\partial_{x}\left[\left(1+x^{2}\right) \partial_{x} \psi\right]+\frac{1}{\sin \theta} \partial_{\theta}\left(\sin \theta \partial_{\theta} \psi\right)+ \\
\frac{(\omega+m x)^{2}}{1+x^{2}} \psi-\frac{m^{2}}{\sin ^{2} \theta} \psi=\mu^{2} \psi
\end{gathered}
$$

This equation clearly separates

$$
\psi(x, \theta)=\Theta(\theta) X(x)
$$

splitting into the pair of ordinary differential equations

$$
\begin{gathered}
\partial_{x}\left[\left(1+x^{2}\right) \partial_{x} X\right]+\frac{(\omega+m x)^{2}}{1+x^{2}} X-\mu^{2} X=\mathcal{K}^{2} X \\
-\frac{1}{\sin \theta} \partial_{\theta}\left(\sin \theta \partial_{\theta} \Theta\right)+\frac{m^{2}}{\sin ^{2} \theta} \Theta=\mathcal{K}^{2} \Theta
\end{gathered}
$$

(we use the same symbol for the total angular momentum as for geodesics). The angular equation is that for the associated Legendre functions, so the separation constant is

$$
\mathcal{K}^{2}=l(l+1), \quad l \geq|m|, \quad l=1,2, \ldots
$$

The radial equation after the change of the variable

$$
x=i \frac{\xi+1}{\xi-1}
$$

reduces to the hypergeometric type equation

$$
\xi^{2}(\xi-1)^{2} \partial_{\xi}^{2} X+\xi(\xi-1)^{2} \partial_{\xi} X-\frac{1}{4}\left(\omega_{+} \xi-\omega_{-}\right)^{2} X-\left(\mathcal{K}^{2}+\mu^{2}\right) \xi X=0,
$$

where $\omega_{ \pm}=\omega \pm i m$. Its general solution reads

$$
X=C_{1} \xi^{\alpha}(1-\xi)^{h} F\left(a_{+}, b_{+} ; c_{+} ; \xi\right)+C_{2} \xi^{-\alpha}(1-\xi)^{h} F\left(a_{-}, b_{-} ; c_{-} ; \xi\right)
$$

with

$$
\alpha=\frac{1}{2}(\omega-i m), \quad h=\frac{1}{2}(1+\lambda), \quad a_{ \pm}=h \pm \omega, \quad b_{ \pm}=h \mp i m, \quad c_{ \pm}=1 \pm 2 \alpha,
$$

and

$$
\lambda=\sqrt{1+4\left(\mathcal{K}^{2}+\mu^{2}-m^{2}\right)} .
$$

When $x \rightarrow \pm \infty$ the argument $\xi$ of the hypergeometric functions goes to 1 , and both diverge as $(1-\xi)^{-\lambda}$ making the solution non-square integrable, unless the series reduces to a polynomial for $a_{ \pm}=h \pm \omega=-n$ with $n$ integer. Choosing $\omega>0$ (in which case $C_{1}=0$ ), this condition reads

$$
\omega=n+\frac{1}{2}+\sqrt{\left(l+\frac{1}{2}\right)^{2}-m^{2}+\mu^{2}} .
$$


One sees that the energy spectrum of the minimally coupled scalar field is discrete both for massive and massless particles. Recall that in the latter case classical geodesics can escape to infinity, but this takes an infinite time. Note that the spectrum is not degenerate with respect to the azimuthal quantum number $m$, which could be expected for lack of spherical symmetry. For $m=0$ we recover from our formula (3.21) the usual result for quantization on $A d S_{2} \times S^{2}$.

For the near horizon limit of the extremal Kerr-(Newman) 38 one finds essentially the same radial equation, but a different angular equation. In that case the separation constant $\mathcal{K}^{2}$ is such that for certain modes $\lambda$ is complex. This corresponds to the continuous spectrum. It was shown in [38] that in this part of the spectrum there are superradiant modes inherited from the Kerr field.

Coming back to the spectrum (3.21), one can see that it is equidistant, which may indicate that it can be obtained purely algebraically. Indeed, the anti-de Sitter group $S L(2, R)$ may be used as a dynamical symmetry. In other terms, one can put the $x, t$ part of the wave equation into the form of a conformal quantum mechanics.

\section{Conformal mechanics}

Let us show that the $R B R^{0}$ geometry generates a modified version of the relativistic conformal mechanics found recently in [7] as a generalization of the de Alfaro-FubiniFurlan non-relativistic model. One starts by multiplying the interval (2.8) par $M^{2}=$ const and rescaling time by $\tau \rightarrow M^{-1} \tau$ to arrive at the metric

$$
d s^{2}=-r^{2} d \tau^{2}+\left(\frac{M}{r}\right)^{2} d r^{2}+M^{2}\left[d \theta^{2}+\sin ^{2} \theta(d \phi+r d \tau / M)^{2}\right] .
$$

The associated Klein-Gordon operator is

$$
-p^{2} \equiv \frac{1}{\sqrt{-g}} p_{\nu} \sqrt{-g} g^{\nu \lambda} p_{\lambda}=M^{-2}\left(-\mathcal{C}+\mathcal{K}^{2}-L^{2}\right)
$$

where

$$
L=-p_{\phi}, \quad \mathcal{K}^{2}=p_{\theta}^{2}+\sin ^{-2} \theta p_{\phi}^{2},
$$

and

$$
\mathcal{C}=L_{0}^{2}-\frac{1}{2}\left(L_{1} L_{-1}+L_{-1} L_{1}\right)
$$

is the Casimir operator for the $\operatorname{sl}(2, R)$ algebra.

Define the Hamiltonian as the time-component $p_{\tau}$ of the four-momentum. The massshell constraint $p^{2}=\mu^{2}$ may be solved to eliminate $p_{\tau}$ in terms of $r, p_{r}$ and the angular quantum numbers $K$ and $L$. Introducing for convenience a new variable

$$
\rho=\frac{2 M}{r^{1 / 2}}
$$

so that the $\mathrm{RBR}^{0}$ metric takes the form

$$
d s^{2}=-\left(\frac{2 M}{\rho}\right)^{4} d \tau^{2}+\left(\frac{2 M}{\rho}\right)^{2} d \rho^{2}+M^{2}\left[d \theta^{2}+\sin ^{2} \theta\left(d \phi+4 M d \tau / \rho^{2}\right)^{2}\right]
$$


we thus obtain the Hamiltonian

$$
H=\left(\frac{2 M}{\rho}\right)^{2}\left\{\sqrt{\mu^{2}+\left(\rho^{2} p_{\rho}^{2}+4 \mathcal{K}^{2}\right) /(2 M)^{2}}-L / M\right\} .
$$

Following [7] one can rewrite this as

$$
H=\frac{p_{\rho}^{2}}{2 f}+\frac{\mu g}{2 f \rho^{2}}
$$

with

$$
f=\frac{1}{2}\left(\sqrt{\mu^{2}+\left(\rho^{2} p_{\rho}^{2}+4 \mathcal{K}^{2}\right) /(2 M)^{2}}+L / M\right)
$$

where

$$
g=4 M^{2} \mu+4\left(\mathcal{K}^{2}-L^{2}\right) / \mu .
$$

This is precisely the expression found in [7] for a charged particle in the Bertotti-Robinson spacetime and the corresponding electromagnetic field. In our case the particle is uncharged, but comparing with [7] one can see that now the normalized azimuthal momentum $L / M$ enters $f$ in the same way as the electric charge in [7]. This is the only modification to the usual Bertotti-Robinson case which is introduced by the RBR geometry. The DFF mechanics of [9] is recovered in the non-relativistic limit $M \rightarrow \infty, L / M \rightarrow \mu$ keeping $g$ fixed. Then $f \rightarrow \mu$ and we get

$$
H=\frac{p_{\rho}^{2}}{2 \mu}+\frac{g}{2 \rho^{2}} .
$$

From the relativistic conformal mechanics point of view, the three generators of the $S L(2, R)$ symmetry are

$$
H=i M^{-1} L_{1}^{\prime}, \quad D=2 i L_{0}^{\prime}, \quad K=i M L_{-1}^{\prime},
$$

where the reduced generators $L_{n}^{\prime}$ are obtained from the Killing vectors (2.17) by eliminating $p_{\tau}=H\left(\rho, p_{\rho}\right)$ and the conjugate variable $\tau=0$. The generators of dilations $D$ and of special conformal transformations $K$ are given by

$$
D=\rho p_{\rho}, \quad K=\frac{1}{2} f \rho^{2} .
$$

These are the spectrum generating operators which, acting on the eigenstates of $H$, produce other eigenstates. It is easy to show that the action of the exponential operator $\exp (i \alpha D)$ on a state with an energy $\omega$ gives an eigenstate with the energy $\exp (2 \alpha) \omega$, this means that the energy spectrum is continuous. In addition, the lowest energy eigenstate is not normalizable. This may seem strange in view of the result which we obtained in the previous section for the Klein-Gordon equation. Note, however, that we have used a different coordinate patch, namely, $\mathrm{RBR}^{+}$. The crucial difference is that this patch does not have a horizon. The explanation comes in exactly the same way as for the ordinary Bertotti-Robinson geometry [10]. 
First recall that already in [9] it was argued that one can redefine the Hamiltonian in such a way that the spectrum becomes discrete. Any linear combination of the three generators $H, D, K$ can be used as an evolution operator since all of them generate transformations leaving the action invariant. The particular combination

$$
H^{\prime}=H+K
$$

was suggested in [9] as a 'better' Hamiltonian with discrete spectrum. Indeed, (4.14) amounts to adding a quadratic potential to $H$. Gibbons and Townsend [10] noted that, in the context of the BR geometry, the transition from the (relativistic version of) $H$ to $H^{\prime}$ is equivalent to a redefinition of the Hamiltonian with respect to the time variable on the globally defined coordinate patch (see also [23]). We shall now verify that this remains true for the RBR geometry as well, thus explaining why we have obtained a discrete spectrum for a test scalar field on the background of $\mathrm{AdS}^{+}$, and show that the $\operatorname{sl}(2, R)$ algebra expressed in $t, x$ coordinates acts indeed as a spectrum generating algebra.

We expect that the raising and lowering spectrum generating operators $\mathcal{L}_{ \pm 1}$ will be hermitean conjugate to each other, and we wish to choose the hamiltonian? as $\mathcal{H}=\mathcal{L}_{0}=$ $i \partial_{t}$. Then the $\operatorname{sl}(2, R)$ algebra can be recast into the following form

$$
\begin{aligned}
\mathcal{L}_{0} & =\frac{i}{2}\left(L_{1}+L_{-1}\right), \\
\mathcal{L}_{ \pm 1} & =\frac{i}{2}\left(L_{1}-L_{-1}\right) \mp L_{0},
\end{aligned}
$$

so that again

$$
\left[\mathcal{L}_{n}, \mathcal{L}_{m}\right]=(n-m) \mathcal{L}_{n+m}
$$

but now (the $L_{n}$ being antihermitean) $\mathcal{L}_{0}$ is hermitean $\mathcal{L}_{0}^{+}=\mathcal{L}_{0}$, while $\mathcal{L}_{1}^{+}=\mathcal{L}_{-1}$.

Let us introduce for $\mathrm{AdS}^{0}$ the 'light cone' coordinates $t^{ \pm}$:

$$
\tau=\frac{1}{2}\left(t^{+}+t^{-}\right), \quad r=2\left(t^{+}-t^{-}\right)^{-1}
$$

Then passing from $\mathrm{AdS}^{0}$ to $\mathrm{AdS}^{+}$amounts to the following transformation

$$
t^{ \pm}=\tan u^{ \pm}
$$

and it can be checked that our previous $\mathrm{AdS}^{+}$coordinates are related to these as

$$
t=u^{+}+u^{-}, \quad \arctan \left(x^{-1}\right)=u^{+}-u^{-} .
$$

For the angular part we have

$$
\phi=\varphi+\ln \left|\frac{\cos u^{+}}{\cos u^{-}}\right|
$$

so the entire metric reads

$$
d s^{2}=-\frac{4 d u^{+} d u^{-}}{\sin ^{2}\left(u^{+}-u^{-}\right)}+d \theta^{2}+\sin ^{2} \theta\left(d \varphi+\cot \left(u^{+}-u^{-}\right)\left(d u^{+}+d u^{-}\right)\right)^{2} .
$$

\footnotetext{
${ }^{2}$ We use in the following units such that $M=1$.
} 
In the new variables the above generators take the more symmetric form

$$
\begin{aligned}
\mathcal{L}_{0} & =\frac{i}{2}\left(\partial_{+}+\partial_{-}\right) \\
\mathcal{L}_{ \pm 1} & =\frac{i}{2}\left[\mathrm{e}^{ \pm 2 i u^{+}}\left(\partial_{+} \mp i \partial_{\varphi}\right)+\mathrm{e}^{ \pm 2 i u^{-}}\left(\partial_{-} \pm i \partial_{\varphi}\right)\right]
\end{aligned}
$$

with

$$
\partial_{ \pm} \equiv \partial_{u^{ \pm}}=\partial_{t} \mp\left(1+x^{2}\right) \partial_{x}
$$

Restricting them to modes depending on $\varphi$ as $\mathrm{e}^{i m \varphi}$, one obtains

$$
\mathcal{L}_{ \pm 1}^{(m)}=\frac{i}{2}\left[\mathrm{e}^{ \pm 2 i u^{+}}\left(\partial_{+} \pm m\right)+\mathrm{e}^{ \pm 2 i u^{-}}\left(\partial_{-} \mp m\right)\right] .
$$

To make contact with our previous treatment of the Klein-Gordon equation let us write the $\operatorname{sl}(2, R)$ Casimir operator:

$$
\begin{aligned}
& \mathcal{C} \equiv \mathcal{L}_{0}\left(\mathcal{L}_{0}-1\right)-\mathcal{L}_{-1}^{(m)} \mathcal{L}_{1}^{(m)}= \\
& -\sin ^{2}\left(u^{+}-u^{-}\right)\left(\partial_{+} \partial_{-}+m^{2}\right)+\frac{i m}{2} \sin \left[2\left(u^{+}-u^{-}\right)\right]\left(\partial_{+}+\partial_{-}\right) .
\end{aligned}
$$

For eigenmodes of $\mathcal{L}_{0}=i \partial_{t}$ with eigenvalue $\omega$, this is (according to (4.23)) identical to the radial Klein-Gordon operator

$$
\mathcal{C}=\partial_{x}\left(1+x^{2}\right) \partial_{x}+\frac{(\omega+m x)^{2}}{1+x^{2}}-m^{2}
$$

with non-negative eigenvalues $\left(\lambda^{2}-1\right) / 4$ (where $\lambda$ is given in $(3.20)$ ).

The representation of the spectrum generating algebra now can be found in a standard way. Assuming (without loss of generality) $\omega>0$, the ground state of $\mathcal{L}_{0}$ is annihilated by $\mathcal{L}_{1}$ :

$$
\mathcal{L}_{0} \phi_{0}=\omega_{0} \phi_{0}, \quad \mathcal{L}_{1} \phi_{0}=0
$$

the solution being

$$
\phi_{0}=\xi^{(i m-h) / 2}(1-\xi)^{h}, \quad \xi=\mathrm{e}^{2 i\left(u^{+}-u^{-}\right)}, \quad \omega_{0}=h,
$$

where $h=(1+\lambda) / 2$ is the conformal weight. As follows from the commutation relations (4.16), the operator $\mathcal{L}_{-1}^{(m)}$ acts as a raising operator in the space of the eigenvectors of $\mathcal{L}_{0}$, shifting the eigenvalue by unity, and consequently

$$
\omega_{n}=h+n .
$$

Acting $n$ times by

$$
\mathcal{L}_{-1}^{(m)}=\left(\xi^{-1 / 2}+\xi^{1 / 2}\right) \mathcal{L}_{0} / 2-\left(\xi^{-1 / 2}-\xi^{1 / 2}\right)\left(\xi \partial_{\xi}+i m / 2\right),
$$

we find the following expression for the $n$-th eigenfunction

$$
\phi_{n}=\xi^{(i m-h-n) / 2}(1-\xi)^{h} F(-n, h+i m ; 1-h-n+i m ; \xi),
$$


where the hypergeometric function with one of the first two indices equal to a negative integer is a polynomial of degree $n$. This is exactly our previous solution (3.18) with $C_{1}=0$. In checking this formula it is helpful to derive first the following operator identities

$$
\begin{aligned}
\mathcal{L}_{-1}^{(m)} \xi^{-i m / 2} & =\xi^{-i m / 2} \mathcal{L}_{-1}^{(0)} \\
{\left[\xi \partial_{\xi},\left(\xi^{-1 / 2}-\xi^{1 / 2}\right)^{-\omega}\right] } & =\frac{\omega}{2} \frac{\left(\xi^{-1 / 2}+\xi^{1 / 2}\right)}{\left(\xi^{-1 / 2}-\xi^{1 / 2}\right)}\left(\xi^{-1 / 2}-\xi^{1 / 2}\right)^{-\omega}
\end{aligned}
$$

and to use the recurrence relation between hypergeometric functions

$$
\frac{d}{d \xi}\left(\xi^{c-1}(1-\xi)^{b-c+1} F(a, b ; c ; \xi)\right)=(c-1) \xi^{c-2}(1-\xi)^{b-c} F(a-1, b ; c-1 ; \xi),
$$

arriving at the recurrence between eigenfuctions

$$
\mathcal{L}_{-1}^{(m)} \phi_{n}=(h+n-i m) \phi_{n+1} .
$$

If one tries to put the $R B R^{+}$problem into the form of a relativistic DFF conformal mechanics, the result can not be expressed in algebraic form. For a discussion of this point in the ordinary BR case see [47].

\section{$5 \quad$ Asymptotic symmetry}

Now let us explore asymptotic symmetries, which are, by definition, the coordinate transformations leaving the metric invariant as $r \rightarrow \infty$. To find the corresponding generators $\mathcal{K}$ one has to solve the Killing equations

$$
g_{\mu \nu, \lambda} \mathcal{K}^{\lambda}+g_{\mu \lambda} \mathcal{K}_{, \nu}^{\lambda}+g_{\nu \lambda} \mathcal{K}_{, \mu}^{\lambda}=0
$$

assuming a suitable falloff for the metric near the boundary. For BR these are given by the asymptotic symmetries of $A d S_{2}$ together with the exact symmetry $S O(3)$ of the full spacetime. The former were discussed in a number of papers [25, 27, 29, 26, 30]. One assumes in the $A d S_{2}$ sector (for $A d S^{0}$ patch)

$$
g_{\tau \tau}=-r^{2}+O(1), \quad g_{r r}=1 / r^{2}+O\left(1 / r^{4}\right), \quad g_{r \tau}=O\left(1 / r^{3}\right) .
$$

The solution of (5.1) then reads

$$
\mathcal{K}=\left(\epsilon(\tau)+\frac{1}{2 r^{2}} \epsilon^{\prime \prime}(\tau)\right) \partial_{\tau}-r \epsilon^{\prime}(\tau) \partial_{r}
$$

where $\epsilon(\tau)$ is an arbitrary (infinitesimal) real function, and primes denote derivatives over

$\tau$. These transformations generate the Virasoro algebra. Indeed, expanding $\epsilon(\tau)$ in the Laurent series

$$
\epsilon(\tau)=\sum_{-\infty}^{\infty} \epsilon_{n} \tau^{(1-n)}
$$


we can write

$$
\mathcal{K}=\sum_{-\infty}^{\infty} \epsilon_{n} L_{n}
$$

where $\epsilon_{n}$ are arbitrary real numbers. The infinite set of generators

$$
L_{n}=\tau^{-n}\left[\left(\tau+\frac{n(n-1)}{2 r^{2} \tau}\right) \partial_{\tau}+(n-1) r \partial_{r}\right],
$$

satisfies the Virasoro algebra without a central charge

$$
\left[L_{n}, L_{m}\right]=(n-m) L_{n+m}
$$

up to terms $O\left(r^{-4}\right)$.

Now let us turn to the RBR spacetime, also choosing the $A d S^{0}$ patch. Actually we shall consider the more general class of metrics

$$
d s^{2}=F(\theta)\left(-r^{2} d \tau^{2}+\frac{d r^{2}}{r^{2}}+d \theta^{2}\right)+\frac{\sin ^{2} \theta}{F(\theta)}(d \phi+\gamma r d \tau)^{2},
$$

( $\gamma$ constant), which includes the extreme Kerr-Newman near-horizon metric [38] as well as the near-horizon metric for extreme rotating dilaton-axion black holes with NUT charge 45. Irrespective of the factor $F(\theta)$, the $S L(2, R) \times U(1)$ symmetry is enlarged to an infinite-dimensional symmetry at the boundary $r \rightarrow \infty$ as well. We have to solve the Killing equations (5.1) assuming the following behavior for the metric as $r \rightarrow \infty$ :

$$
\begin{gathered}
g_{\tau \tau}=-r^{2}\left(F-\gamma^{2} F^{-1} \sin ^{2} \theta\right)+O(1), \quad g_{r r}=F / r^{2}+O\left(1 / r^{4}\right), \\
g_{\phi \phi}=F^{-1} \sin ^{2} \theta+O\left(1 / r^{2}\right), \quad g_{\tau \phi}=\gamma r F^{-1} \sin ^{2} \theta+O(1 / r), \\
g_{\theta \theta}=F+O\left(1 / r^{2}\right), \quad g_{r \tau}=O\left(1 / r^{3}\right), \quad g_{r \phi}=O\left(1 / r^{3}\right),
\end{gathered}
$$

together with suitable conditions for $g_{\theta \phi}, g_{\theta r}, g_{\theta \tau}$. The solution reads

$$
\mathcal{K}=\left(\epsilon(\tau)+\frac{1}{2 r^{2}} \epsilon^{\prime \prime}(\tau)\right) \partial_{\tau}-r \epsilon^{\prime}(\tau) \partial_{r}+\left(k-\gamma \frac{\epsilon^{\prime \prime}(\tau)}{r}\right) \partial_{\phi}
$$

where $k$ is an arbitrary constant. The $k$-term decouples, it generates an exact $U(1)$ symmetry of the bulk spacetime: $L_{\phi}=\partial_{\phi}$. The remaining transformations form again the Virasoro algebra. Carrying out a Laurent expansion one finds

$$
L_{n}=\tau^{-n}\left[\left(t+\frac{n(n-1)}{2 r^{2} \tau}\right) \partial_{\tau}+r(n-1) \partial_{r}-\gamma \frac{n(n-1)}{r \tau} \partial_{\phi}\right]
$$

Note the non-trivial mixing with $\partial_{\phi}$ due to the fact that we no longer deal with the direct product of $A d S_{2}$ and a sphere. The $\operatorname{sl}(2, R)$ subalgebra of the Virasoro algebra coincides with the exact symmetry of RBR exhibited in (2.17). Further work is needed to find out whether the representations of this algebra in terms of asymptotic spacetime deformations acquire a central charge in the sense of Brown and Henneaux [35]. 


\section{Concluding remarks}

Our results suggest that the set of geometries $A d S_{n} \times K$ with compact $K$ relevant for holography can be extended to manifolds which do not have the structure of a direct product. Several examples of such geometries are provided as the near horizon limits of various rotating black holes in four dimensions. Such manifolds have the isometry $S L(2, R) \times U(1)$ and they do not split into $A d S_{2} \times K$ even asymptotically.

We have constructed explicitly conformal mechanics associated to $R B R^{0}$ as a modified relativistic De Alfaro-Fubini-Furlan model in which the angular momentum plays the role of the electric charge. We also checked that the transition to $R B R^{+}$, similarly to the case of $A d S_{2} \times S^{2}$, redefines the time variable in such a way that the new Hamiltonian has a discrete spectrum bounded from below. The associated $S L(2, R)$ symmetry is naturally extended to the Witt algebra which is realized as an asymptotic symmetry of the fourdimensional spacetime.

It is worth noting that although the conformal boundary is singular in the rotating case, the asymptotic symmetry still contains the Virasoro algebra. So in this respect the only difference with the case of the factorizable geometry $A d S_{2} \times S^{2}$ whose boundary is non-singular is that the action of the Virasoro generators cannot be separated from the action of symmetries on $K$. This is valid both for our new rotating BR spacetime, and for the previously considered extremal $\operatorname{Kerr}(-N e w m a n)$ throat. We intend to discuss the issue of the central charge in a separate paper.

Our final remark concerns supersymmetry. Rotation breaks the supersymmetry of $A d S_{2} \times S^{2}$, and our new solution is not supersymmetric from the $N=4, d=4$ supergravity point of view. However, the isometry group $A d S_{2} \times U(1)$ allows for a superextension $S U(1,1 \mid 1)$. It remains to be clarified whether supersymmetry still plays any role in our case.

\section{Acknowledgements}

DG is grateful to LAPTH Annecy for hospitality and to CNRS for support which made possible this collaboration. His work was also supported in part by the RFBR grant 00-02-16306. 


\section{References}

[1] J. M. Maldacena, Adv. Theor. Math. Phys. 2 (1998) 231;

S. S. Gubser, I. R. Klebanov and A. M. Polyakov, Phys. Lett. B428 (1998) 105;

O. Aharony, S. S. Gubser, J. M. Maldacena, H. Ooguri and Y. Oz, Phys. Rept. 323 (2000) 183.

[2] G. Gibbons and C. M. Hull, Phys. Lett. B109 (1982) 190.

[3] K. P. Tod, Phys. Lett. B121 (1983) 241.

[4] H. J. Boonstra, B. Peeters, and K. Skenderis, Nucl. Phys. B533 (1998) 127.

[5] D. Youm, Phys. Rev. D 60 (1999) 064016; Nucl. Phys. B573 (2000) 257.

[6] M. Cadoni, P. Carta, M. Cavaglià, S. Mignemi, Conformal dynamics of 0-branes, hep-th/0105113.

[7] P. Claus, M. Derix, R. Kallosh, J. Kumar, P.K. Townsend and A. Van Proeyen, Phys. Rev. Lett. 81 (1998) 4553.

[8] R. Kallosh, Black holes, branes and superconformal symmetry, hep-th/9901095; Black holes and quantum mechanics, hep-th/9902007.

[9] V. de Alfaro, S. Fubini and G. Furlan, Nuovo Cim. 34A, (1976) 569.

[10] G. W. Gibbons and P. K. Townsend, Phys. Lett. B454 (1999) 187.

[11] J. Michelson and A. Strominger, JHEP 9909 (1999) 005.

[12] J.A. de Azcárraga, J.M. Izquierdo, J.C. Pérez-Bueno and P.K. Townsend, Phys. Rev. D 59 (1999) 084015.

[13] V. Akulov and M. Kudinov, Phys. Lett. B4260 (1999) 365.

[14] N. Wyllard, J. Math. Phys. 41 (2000) 2826.

[15] E. Bergshoeff and M. Vasiliev, Int. Journ. Mod. Phys. A 10 (1995) 3477.

[16] J. Kumar, JHEP 9904 (1999) 006.

[17] J. Kumar, JHEP 0005 (2000) 035.

[18] B. Rai, Dynamics of $A d S_{2}$ and enlargement of $S L(2, R)$ to $c=1$ 'cut-off' Virasoro algebra, hep-th/0104142.

[19] M. Cadoni, P. Carta, and D. Klemm, Phys. Lett. B503 (2001) 205.

[20] J. Maldacena and A. Strominger, Phys. Rev. D 56 (1997) 4975.

[21] J. Maldacena, J. Michelson, and A. Strominger, JHEP 03 (1999) 011. 
[22] J. Michelson, Phys. Rev. D 57 (1998) 1092.

[23] R. Britto-Pacumio, J. Michelson, A. Strominger and A. Volovich, Lectures on superconformal quantum mechanics and multi-black hole moduli space, hep-th/9911066.

[24] A. Strominger, JHEP 01 (1999) 007.

[25] M. Cadoni and S. Mignemi, Phys. Rev.D 59 (1999) 081501; Nucl. Phys. B557 (1999) 165.

[26] J. Navarro-Salas and P. Navarro, Nucl. Phys. B579 (2000) 250.

[27] M. Cadoni and S. Mignemi, Phys. Lett. B490 (2000) 131.

[28] M. Cadoni, Phys. Rev. D 60 (1999) 084016.

[29] M. Cadoni. P. Carta and S. Mignemi, Phys. Rev. D 62 (2000) 086002.

[30] M. Cadoni and M. Cavaglià, Phys. Lett. B499 (2001) 315; Phys. Rev. D 63 (2001) 084024.

[31] M. Cadoni, P. Carta, D. Klemm, S. Mignemi, Phys. Rev. D 63 (2001) 125021.

[32] M. Caldarelli, G. Catelani and L. Vanzo, JHEP 0010 (2000) 005.

[33] G. Catelani and L. Vanzo, On the $\sqrt{2}$ puzzle in $A d S_{2} / C F T_{1}$, hep-th/0009186.

[34] M. Cadoni and P. Carta, Mod. Phys. Lett. A16 (2001) 171.

[35] J. D. Brown and M. Henneaux, Comm. Math Phys. 104 (1986) 207.

[36] H. Terashima, Phys. Rev. D 64 (2001) 064016.

[37] A. Strominger, JHEP 02 (1998) 009.

[38] J. Bardeen and G.T. Horowitz, Phys. Rev. D 60 (1999) 104030.

[39] O. B. Zaslavsky, Class. Quant. Grav. 15 (1998) 3251.

[40] M. Cvetič and F. Larsen, Nucl. Phys. B531 (1998) 239; Phys. Rev. Lett. 82 (1998) 484.

[41] D. Berman, Aspects of holography and rotating AdS black holes, hep-th/0002235.

[42] D. S. Berman and M. K. Parikh, Phys. Lett. B463 (1999) 168.

[43] S. W. Hawking, C. J. Hunter, and M. M. Taylor-Robinson, Phys. Rev. D59 (1999) 064005.

[44] M. M. Caldarelli, D. Klemm, and W. Sabra, JHEP 0105 (2001) 014.

[45] Gérard Clément and Dmitri Gal'tsov, Phys. Rev. D 63 (2001) 124011. 
[46] D. V. Gal'tsov and O. V. Kechkin, Phys. Rev. D 50 (1994) 7394; Hidden symmetries in dilaton-axion gravity", in "Geometry and Integrable models", P.N. Pyatov and S.N. Solodukhin (Eds.), World Scientific, Singapore 1996.

[47] S. Mignemi, Black holes and conformal mechanics, hep-th/0104175. 